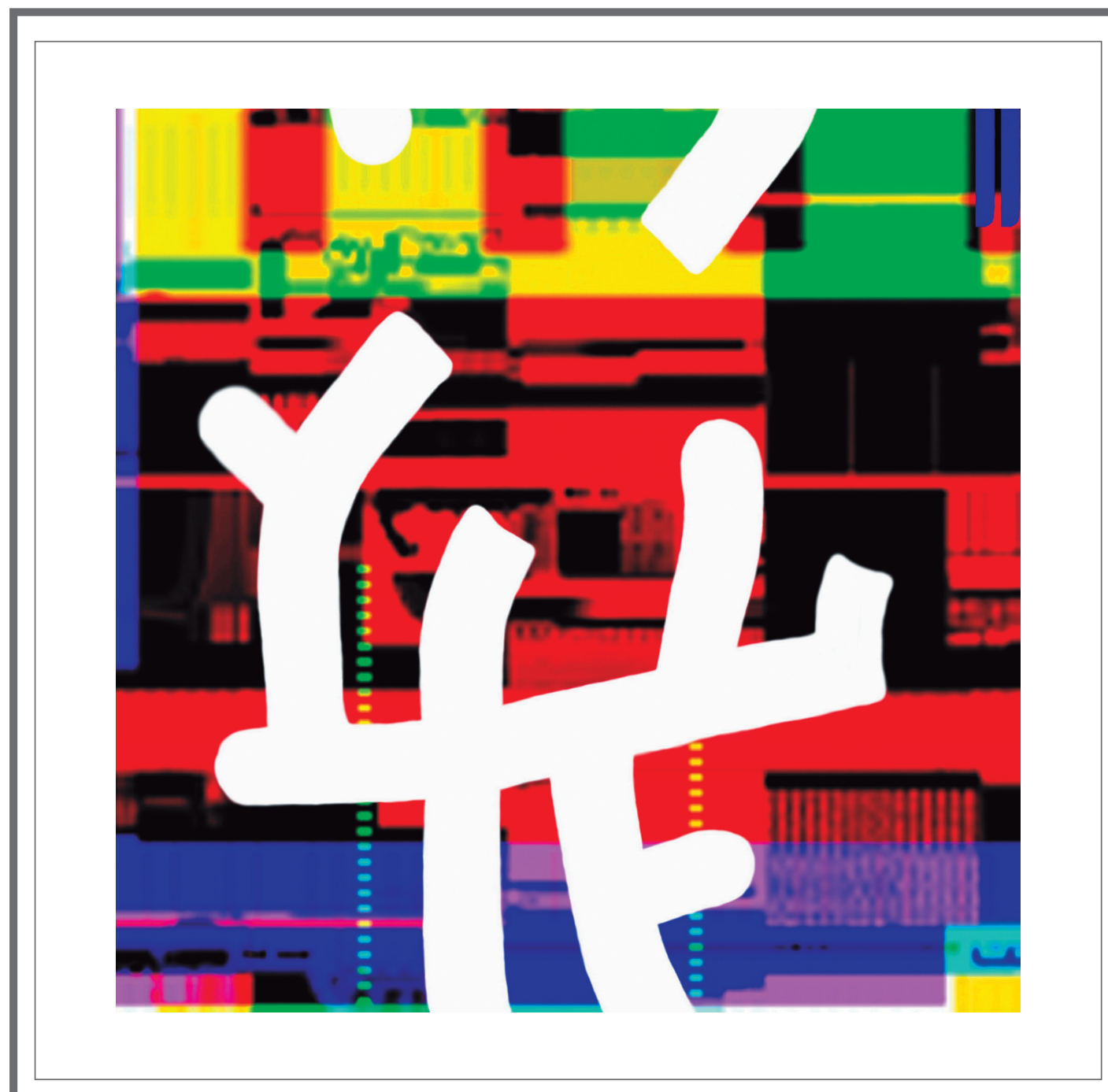

(Arte)

Estampa Digital

Arquitectura 10 P.M.

Titulo:

Arquitectura Neón 


\section{Autor:}

Luis Fernando Luna

Técnica:

Impresión Digital Giclée sobre papel de Algodón 255 gr.

Medida: 40 X $40 \mathrm{~cm}$ (Estampa 30 X $30 \mathrm{~cm}$ )

Edición de 25 ejemplares 


\title{
FACTORES SOCIO AMBIENTALES Y PERFIL DE MORBILIDAD DE LOS MENORES DE 12 AÑOS EN EL DEPARTAMENTO DE SANTANDER
}

\author{
SOCIO ENVIRONMENTAL FACTORS AND MORBIDITY PROFILE OF \\ CHILDREN UNDER 12 YEARS IN THE DEPARTMENT OF SANTANDER
}

Adelaida Manquián Tejos ${ }^{(l)}$

\begin{abstract}
Resumen:
Se sabe que la población infantil es el grupo poblacional más susceptible al impacto del medio ambiente, razón por la cual varias enfermedades que afectan a los niños, entre ellas las enfermedades diarreicas y gastrointestinales, pueden atribuirse a factores ambientales modificables tales como pobreza, agua no apta para el consumo humano, condiciones sanitarias inadecuadas y alimentos contaminados. En Santander, tales enfermedades se ubican entre las principales causas de consulta en todos los niveles de atención de los niños menores de 12 años. Este documento muestra los resultados de un estudio diagnóstico $\mathrm{y}$, aunque corresponden a una mirada parcial del problema pretenden mostrar el entorno socio ambiental en el cual crecen los niños. Los indicadores seleccionados, muestran que existe una distribución inequitativa de los recursos en el Departamento, con alto porcentaje de pobres, falta de saneamiento básico y contaminación de alimentos, factores que probablemente contribuyen a mantener la alta prevalencia de estas enfermedades.
\end{abstract}

Palabras clave: condiciones sanitarias, nivel de ingreso, diarrea, enfermedades gastrointestinales.

\begin{abstract}
It is known that the child population is the most susceptible population group to the impact of the environment, which is why several diseases affecting children, including diarrhea and gastrointestinal diseases can be attributed to modifiable environmental factors such as poverty, water unfit for human consumption, inadequate sanitation and contaminated food. In Santander, such diseases are among the leading causes of consultation at all levels of care for children under 12 years. This paper shows the results of a diagnostic study and even corresponds to a partial view of the problem meant to show the socio environment in which children grow up. The selected indicators show that there is an unequal distribution of resources in the Department, with a high percentage of poor sanitation and lack of food contamination, factors that likely contribute to maintaining the high prevalence of these diseases.
\end{abstract}

Keywords: health status, income level, diarrhea, gastrointestinal diseases.

1 Obstetra, Magíster en Nutrición Humana, Doctora en Medio Ambiente. Docente investigadora Facultad de Química Ambiental, Universidad Santo Tomás. Bucaramanga. 


\section{Introducción}

"La salud ambiental comprende aquellos aspectos de la salud humana, incluida la calidad de vida, que son determinados por factores ambientales físicos, químicos, biológicos, sociales y psicosociales. También se refiere a la teoría y práctica de evaluación, corrección, control y prevención de los factores ambientales que pueden afectar de forma adversa la salud de la presente y futuras generaciones",

Tal concepto fue divulgado por la Organización Mundial de la Salud (OMS) en el año 1993 durante una reunión consultiva celebrada en Sofía, Bulgaria (OMS, 1993; Ordóñez, 2000)

En el mundo, el $24 \%$ de la carga de morbilidad ${ }^{2}$, y el 23\% de todos los fallecimientos ${ }^{3}$ pueden atribuirse o son consecuencia de factores ambientales modificables (PrüssÜstün y Corvalán, 2006). Está demostrado que los niños menores de 14 años son más susceptibles que los adultos al impacto del medio ambiente sobre su salud y calidad de vida (WHO, 2002), debido a factores tales como: sistema inmunitario y mecanismos de detoxificación inmaduros o en desarrollo (Chelala, 1999; Pond et al., 2007; Valent et al. 2004), estimándose que un 36\% de las muertes en esas edades pueden atribuirse a problemas ambientales y, de todas las enfermedades que contribuyen a la carga de morbilidad, la diarrea ocupa el primer lugar con el 29\% (Prüss-Üstün y Corvalán, 2006).

En América Latina se calcula que alrededor de 77.600 niños menores de 5 años mueren anualmente por enfermedad diarreica debida al consumo de agua no apta, a alimentos contaminados y a malos hábitos de higiene (OMS, 2009). En Colombia, en la década 1993 y 2003 se registraron 5 millones de casos con diarrea por causa hídrica con una tasa de mortalidad igual a 5,6 (x100 mil niños). La población más afectada son los pobres (Ramírez, Osorio y Parra, 2007, p. 77).

Todos estos factores se multiplican en las regiones empobrecidas (OMS, 2002; EHP, 2009) y en los sectores rurales:

"alrededor de 103,2 millones de habitantes latinoamericanos (50\% rurales y $10 \%$ urbanos) no tienen acceso a servicios de saneamiento y solamente $13,7 \%$ de las aguas servidas recogidas por los sistemas de alcantarillado son tratadas antes de ser vertidas y, aproximadamente, $41 \%$ de las viviendas con conexiones domiciliarias reciben agua desinfectada. Más de un $60 \%$ de los sistemas de abastecimiento de agua operan en forma intermitente, creando el riesgo de contraer diarrea y otras enfermedades transmitidas por el agua a unos 219 millones de personas" (OMS, 2009).

2 Carga de morbilidad: años de vida sana perdidos. La carga de morbilidad relacionada con el medio ambiente se mide en años de vida ajustados en función de la discapacidad (AVAD), una medida ponderada de la mortalidad, la morbilidad y la discapacidad.

3 Mortalidad prematura 
Mejorar la calidad del agua es una de las medidas que tiene impactantes efectos sobre la salud y supervivencia de los niños y adolescentes (Valent et al., 2004; WHO, 2004; WHO, 2010), reportándose que,

"los beneficios económicos de las inversiones realizadas para mejorar las condiciones sanitarias de la población, son aproximadamente ocho veces superiores a los costos, estos beneficios incluyen aumentos de la productividad económica, reducción de los costos de la atención sanitaria y de los años de vida sana perdidos; a su vez, los mejores comportamientos de higiene contribuyen a romper el ciclo general de contaminación de masas de agua por patógenos fecalesorales, lo cual conlleva beneficios para la salud, la reducción de la pobreza, el bienestar y el desarrollo económico" (Prüss-Üstün y Corvalán, 2006).

En Santander las autoridades reconocen que

"los indicadores de continuidad y calidad del suministro de agua son totalmente insuficientes, en especial en la época seca en la que municipios como Vélez, deben racionar el agua a 1 hora/día", además reconocen que "en materia de tratamiento de aguas residuales, los indicadores son alarmantes, pues solamente un $10 \%$ de las aguas servidas del departamento tienen algún tipo de tratamiento, y las corrientes hídricas receptoras ven afectada su calidad por los vertimientos de alcantarillados y residuos industriales o agrícolas", (PDR, 2008).

Disminuir la pobreza, aumentar la cobertura de acueducto y alcantarillado y mejorar la calidad de estos servicios fue una de las metas del Plan de Desarrollo Regional (PDR) 2008, meta que se ha mantenido en el nuevo PDR, 2012. Se sabe que mientras no se modifiquen tales factores con soluciones permanentes, los problemas de salud persisten y se exacerban (Mardones, 1994).

El conjunto de estos antecedentes motivaron la presentación de este manuscrito que pretende mostrar las características del entorno ambiental donde crecen los niños de Santander y destacar aquellos factores socio - ambientales reconocidos por la autoridad sanitaria OMS como atribuibles a la frecuencia de diarrea y enfermedades gastrointestinales en los niños.

\section{Metodología}

Se realizó en el año 2011 un estudio de tipo diagnóstico descriptivo a partir de fuentes secundarias, como parte del estado del arte de la línea de investigación en Salud Ambiente implementada en la Facultad de Química Ambiental de la Universidad Santo Tomás. La población objetivo es el Departamento de Santander.

Se realizó una revisión bibliográfica que orientó los criterios de selección y análisis de los indicadores utilizados. Se seleccionaron fuentes de datos oficiales y reportes de investigación y/o técnicos de agencias oficiales nacionales (ministerios) e internacionales (Organización Mundial de la Salud (OMS)). 
Los resultados que se presentan en este documento forman parte del estudio mencionado, toda la información proviene de fuentes secundarias que son citadas en el texto, gráficas y tablas.

Una de las debilidades de este estudio es la carencia de información actualizada, por ejemplo, los datos publicados de salud el año 2010 corresponden al año 2008 y 2009, los datos sociales provienen del censo del año 2005.

\section{Resultados}

\section{Enfermedades gastrointestinales y diarreicas}

Entre los años 2005 y 2007, como se muestra en la Gráfica 1, la mortalidad infantil en Santander, así como el número de defunciones por enfermedad diarreica aguda (EDA) tuvo una tendencia ascendente.

Gráfica 1. Tasa de mortalidad de los menores de 5 años (por 1.000 nacidos vivos) y número de defunciones por EDA entre los años 2005 -2007

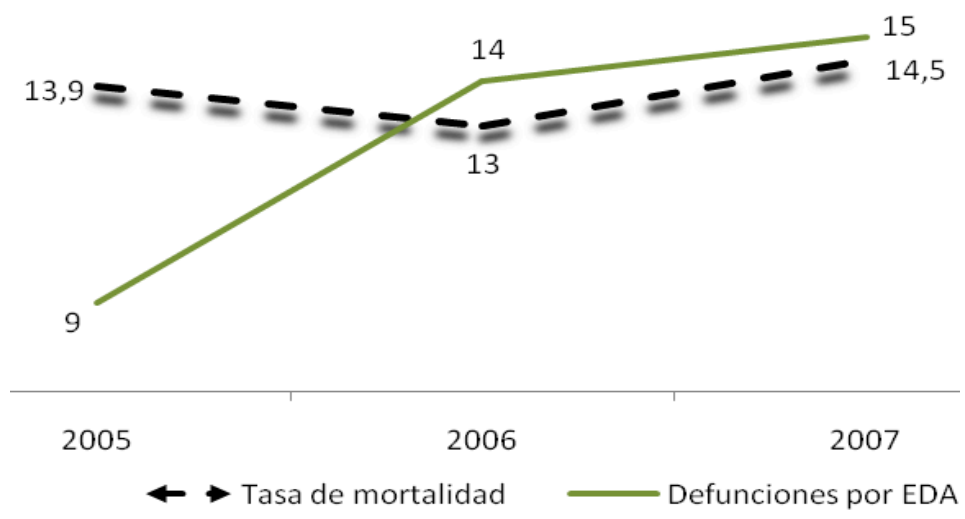

Fuente: SIVIGILA, 2010

De un total de 4.315 .340 consultas realizadas por la población en los diferentes niveles de atención en 20094, 927.826 fueron realizadas por niños menores de 12 años, lo que representa el 21,5\% del total de las consultas (OSPS, 2010).

4 Los últimos datos de morbilidad, disponibles en el Departamento de Santander provienen de la compilación de Registros Individuales de Prestación de Servicios (RIPS) del año 2008, enviados por las Entidades administrativas de planes de beneficiarios EAPB al Observatorio de Salud Pública de Santander. 
Las principales causas de consulta fueron por enfermedades gastrointestinales y respiratorias, sumadas a fiebre de origen desconocido (primera causa de consulta en urgencia y observación), infecciones virales, caries dentales (en consulta externa) y dengue clásico y hemorrágico en los servicios de hospitalización.

La enfermedad diarreica y gastroenteritis de origen infeccioso es la quinta causa de consulta externa de morbilidad y la segunda en los servicios de urgencia y hospitalización. En todos los niveles de atención más del 50\% de estas consultas son realizadas por niños, la excepción es en el nivel de hospitalización, donde el 57,6\% de los hospitalizados por diarrea son niñas. Una mirada retrospectiva muestra que esta tendencia se ha mantenido a través del tiempo. (SSD, 2004; OSPS, 2006; 2007), destacándose que el diagnóstico de diarrea y gastroenteritis en el 80\% de los casos es de presunto origen infeccioso (OSPS, 2007; 2009), situación que como muestran los antecedentes podría atribuirse a factores ambientales modificables.

\section{Crecimiento económico}

De acuerdo con el indicador Producto Interno Bruto (PIB), el Departamento de Santander además de estar entre los de mayor desarrollo (ocupa el cuarto lugar) es uno de los departamentos del país cuyo PIB ha crecido sostenidamente la última década (ver la Gráfica 2) (Cepeda, 2010).

Gráfica 2. Crecimiento del PIB en Santander. Período 2001-2010

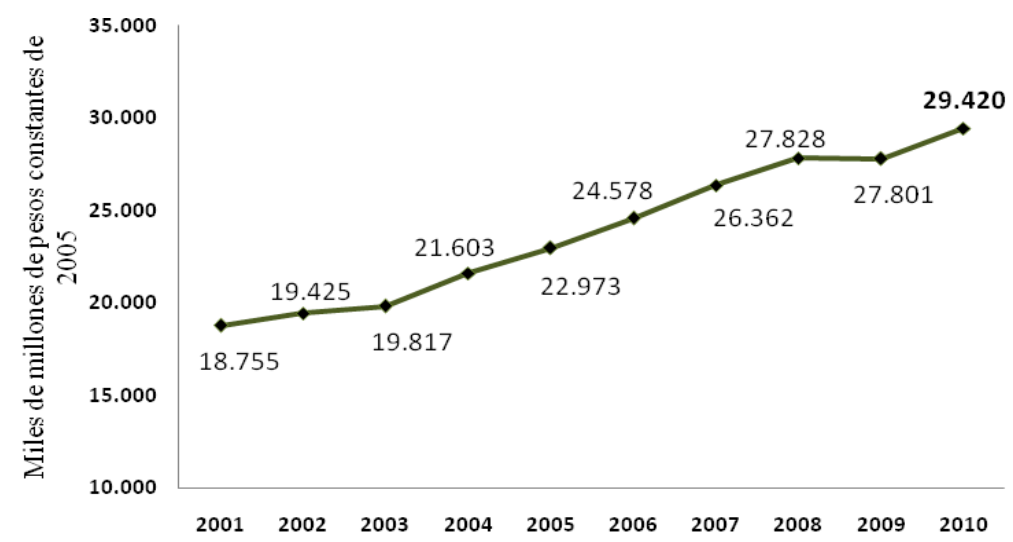

Fuente: PIB, Web, 2012

Entre los años 2003 y 2006 (Gráfica 3) Santander mostró una tasa de incremento de su PIB superior a la de todo el país (ACR, 2008). 
Gráfica 3. Crecimiento del PIB en Colombia y Santander. Período 2003-2006

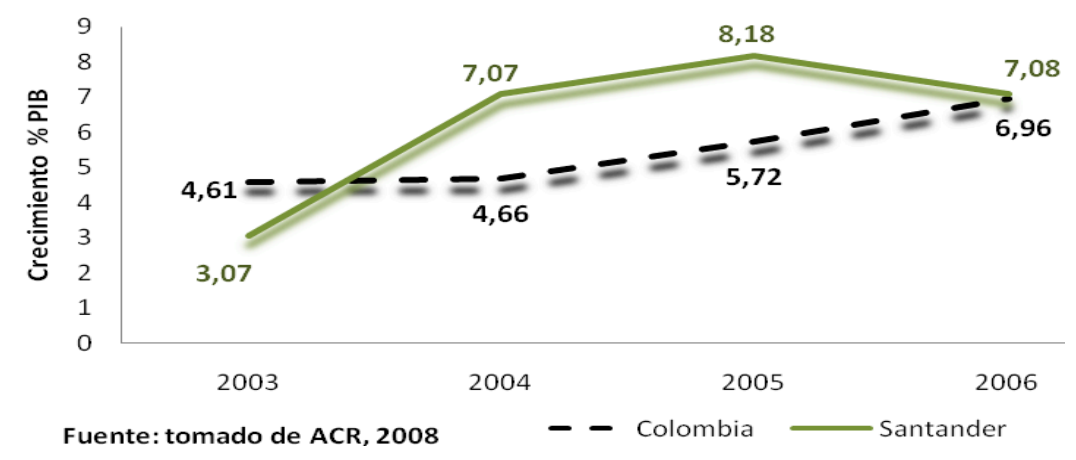

Cepeda (2010) reporta que los sectores de comercio, industria y de servicios empresariales son los que más han aportado al crecimiento de Santander en el período 2000 - 2007. Dentro de la industria, el sector de refinación de petróleo y el de elaboración de productos alimenticios son los que más valor agregado generan.

\section{Nivel de ingreso de la población}

El nivel de ingreso de la población se estudió mediante el comportamiento de cuatro indicadores: ingreso para gastos básicos, necesidades básicas insatisfechas $\left(\mathrm{NBI}^{5}\right)$, extrema pobreza o miseria e índice de condiciones de vida $\left(\mathrm{ICV}^{6}\right)$.

Como se muestra en la Tabla 1, mayoritariamente (entre 62,5 y 85,3\%) la población de todas las provincias declara que sus ingresos no alcanzan para los gastos básicos; sólo para una minoría (menos del 2\%) son más que suficientes.

5 NBI: indicador que define la pobreza para lo cual integra variables de viviendas inadecuadas, con hacinamiento crítico, con servicios inadecuados, con alta dependencia económica y con niños en edad escolar que no asisten a la escuela.

6 ICV, mide: calidad de la vivienda, tamaño y composición del hogar, educación y capital humano y acceso y calidad de los servicios. 
Tabla 1. Porcentajes de las diferentes respuestas a la pregunta sobre los ingresos en las provincias de Santander

\begin{tabular}{lcccc}
\hline \multicolumn{4}{c}{ Ingresos para los gastos básicos } \\
\hline \multicolumn{1}{c}{ Provincias } & Son suficientes & Más que Suficientes & No Alcanzan & Total \\
\hline Mares & 24,7 & 1,6 & 73,7 & 72910 \\
Guanentina & 18,3 & 0,9 & 80,8 & 36020 \\
García Rovira & 14,1 & 0,6 & 85,3 & 19728 \\
Comunera & 17,8 & 0,7 & 81,5 & s24328 \\
Soto & 35,3 & 2,2 & 62,5 & 286206 \\
Vélez & 20,0 & 1,7 & 78,3 & 47946 \\
Santander & 29,2 & 1,8 & 69,0 & 487138 \\
\hline
\end{tabular}

Fuente: Cálculos realizados con datos del censo 2005 (DANE)

El índice NBI muestra que en el Santander 22 de cada 100 personas residentes en él son pobres y, de ellos, la mayoría vive en el sector rural y/o fuera del casco urbano?

La información desagregada según provincias de residencia, muestra que en García Rovira y en Vélez más del 40\% de sus residentes son pobres.

La pobreza es mayor en el sector rural si se compara con el sector urbano, donde el porcentaje de pobres es el doble (Guanentina, García Rovira, Soto y Vélez) y casi el triple (Santander) de lo reportado en el sector urbano, ver la Tabla 2.

7 Cabecera municipal: delimitación geográfica definida por el DANE para fines estadísticos, Corresponde al área geográfica delimitada por el perímetro censal. A su interior se localiza la sede administrativa del municipio, es decir, la Alcaldía. Resto del municipio: área geográfica comprendida entre el Perímetro Censal y el Límite Municipal definido por Ordenanza de la Asamblea Departamental. 
Tabla 2. Porcentaje de personas con NBI en las provincias santandereanas

\begin{tabular}{lccc}
\hline & \multicolumn{3}{c}{ Porcentaje de la Población con NBI } \\
\hline \multicolumn{1}{c}{ Provincias } & Urbano & Rural & Total \\
\hline Mares & 24,9 & 45,7 & 37,3 \\
Guanentina & 17,1 & 45,1 & 36,9 \\
García Rovira & 20,2 & 59,8 & 48,1 \\
Comunera & 23,3 & 44,2 & 37,9 \\
Soto & 16,5 & 36,8 & 27,9 \\
Vélez & 23,7 & 47,7 & 42,3 \\
Santander & 13,4 & 45,4 & 21,9 \\
\hline
\end{tabular}

Fuente: DANE. Datos con base en censo 2005, actualizados a 30 de julio de 2010

126.630 personas residentes en el departamento viven en condiciones paupérrimas o de miseria, lo que representa el 6,6\% del total de la población.

En García Rovira y Vélez de cada 100 residentes más de 10 viven en condiciones de miseria o extrema pobreza (Tabla 3).

Tabla 3. Extrema pobreza en Santander y en cada una de las provincias

\begin{tabular}{lccc}
\hline & \multicolumn{3}{c}{ Extrema pobreza o miseria } \\
\hline Provincias & Poblaciòn total & $\begin{array}{c}\text { Personas en } \\
\text { Miseria }\end{array}$ & $\begin{array}{c}\text { \% del } \\
\text { Total }\end{array}$ \\
\hline Mares & 298.503 & 26.986 & 9 \\
Guanentina & 137.777 & 12.874 & 9,3 \\
García Rovira & 76.730 & 13.030 & 17 \\
Comunera & 96.131 & 8.924 & 9,3 \\
Soto & 1.120 .653 & 39.119 & 3,5 \\
Vélez & 183.466 & 25.697 & 14 \\
Santander & 1.913 .260 & 126.630 & 6,6 \\
\hline
\end{tabular}

Fuente: Datos tomados de Plan de Desarrollo Departamental 2008 - 2011. Santander

Según el ICV, la calidad de vida de la población ha mejorado en el sector urbano no así en el sector rural y los municipios pequeños. Entre el año 2003 y el año 2005 
el ICV de Santander aumentó 1,14 puntos (78 vs. 79,14), los municipios con mejores condiciones de vida en el año 2003 fueron, Floridablanca $(78,6)$, Bucaramanga $(77,6)$ y Girón (76,4); y, el municipio con menor ICV era Jordán $(52,7)$ (ACR, 2008).

La Gráfica 4 muestra el aporte (\%) de cada uno de los indicadores del ICV en Santander, donde se observa que la educación y capital humano es el componente que mayor aporta a este indicador. A pesar de este avance, aún casi 8 de 100 personas mayores de 15 años son analfabetos y un $9,8 \%$ no ha alcanzado ningún nivel educativo (DANE, 2010).

Gráfica 4. ICV de los residentes de Santander

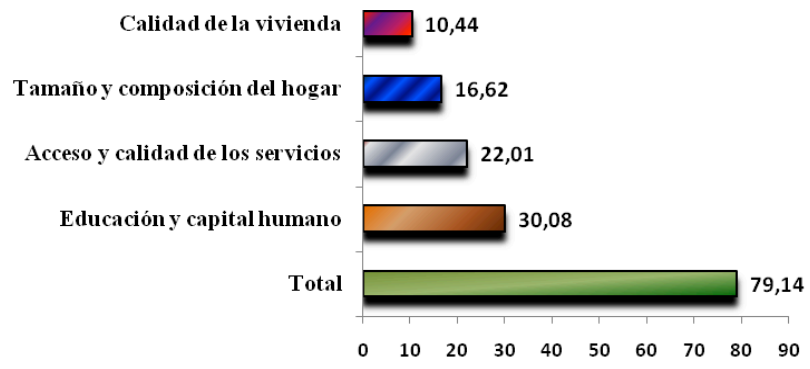

Fuente: datos tomados de ACR, 2008

\section{Condiciones sanitarias de la población}

Para estudiar este factor se seleccionaron los indicadores: origen y calidad del agua, tipo de servicio sanitario, disposición de basuras y calidad de los alimentos.

Al comparar a Colombia con América Latina se observa que, a pesar de que el país ha mejorado, se ubica por debajo de la media regional en varios de estos indicadores. Por ejemplo, en la proporción de la población que utiliza fuentes mejoradas de agua potable, se ubica en el puesto 21 con 92\%, (media regional 96\%) superado por países como Guatemala, país reconocido por sus altos índices de pobreza (OMS, 2010).

Si se compara este indicador con la realidad de Santander la situación parece crítica, en especial en las provincias más vulnerables como García Rovira y Vélez. La disponibilidad de fuentes mejoradas de agua potable en Santander, medida por la disponibilidad de acueducto, asumido que sólo de él proviene agua apta para el consumo humano, muestra que sólo el $82,6 \%$ de la población dispone de este servicio ( $9,4 \%$ menos que Colombia) y de ellos el $2,1 \%$ no la utilizan para su consumo.

García Rovira, Vélez, Comunera son provincias que no superan el 70\%, es decir, más del $30 \%$ de la población residente en estas comunas no cuenta con agua apta para su consumo. 
A pesar de que el acueducto es la fuente de agua para el consumo humano más importante en Santander, es un beneficio que marca brechas de desigualdad entre las provincias, García Rovira, Comunera y Vélez son las que tienen mayor carencia. En la Tabla 4 se muestran la valoración de los diferentes orígenes del agua en las diferentes provincias de Santander.

Tabla 4. Orígenes del agua para consumo en las provincias de Santander

\begin{tabular}{lcccc}
\hline \multicolumn{5}{c}{ Origen del agua para cocinar los alimentos } \\
\hline Provincias & Acueducto & $\begin{array}{c}\text { Río, quebrada, } \\
\text { manantial, } \\
\text { nacimiento }\end{array}$ & $\begin{array}{c}\text { Pozo con o sin } \\
\text { bomba }\end{array}$ & Otro(*) \\
\hline Mares & 81,2 & 5,4 & 9,0 & 2,0 \\
Guanentina & 75,2 & 13,9 & 9,7 & 0,9 \\
García Rovira & 57,4 & 36,6 & 4,7 & 0,6 \\
Comunera & 64,9 & 16,6 & 16,5 & 1,0 \\
Soto & 87,0 & 5,4 & 4,0 & 1,8 \\
Vélez & 62,2 & 15,1 & 19,3 & 2,4 \\
Santander & 80,5 & 8,8 & 7,3 & 1,7 \\
\hline
\end{tabular}

(*) Incluye: agua lluvia, pila pública, carro tanque y agua embotellada

Fuente: Cálculos realizados con base a datos del censo 2005 (DANE)

El 36,6\% de las viviendas de García Rovira obtienen el agua para el consumo humano de los ríos, quebradas, manantiales o nacimientos.

Con respecto a la clasificación que realiza la Secretaría de Salud Ambiental del Departamento, el agua no es adecuada para su consumo la mayor parte del tiempo. Lo esperado es que cada vez que se controle la calidad del agua su clasificación para el consumo humano sea sin riesgo.

Aunque todas las provincias muestran índices de alto riesgo superiores al 13\%, las provincias más propicias a consumir agua contaminada son precisamente las más empobrecidas del departamento - Vélez y García Rovira.

Llama la atención los bajos porcentajes de la clasificación 'Sin Riesgo' para todas las provincias. En particular, Vélez, durante el año 2008, en ningún momento tuvo agua en esta categoría y Soto, la provincia más poblada del departamento, sólo el $7 \%$ de las veces se ubicó en esta categoría. Los porcentajes obtenidos durante el año 2008 en cada una de las clasificaciones de la calidad del agua en las provincias de Santander se reportan en la Tabla 5. 
Tabla 5. Clasificación del Índice de Riesgo de Calidad del Agua para Consumo Humano en Santander y sus provincias. Año 2008

\begin{tabular}{lcccc}
\hline \multicolumn{1}{c}{ Provincias } & $\begin{array}{c}\text { Alto Riesgo } \\
(\%)\end{array}$ & $\begin{array}{c}\text { Riesgo Medio } \\
(\%)\end{array}$ & $\begin{array}{c}\text { Riesgo Bajo } \\
(\%)\end{array}$ & $\begin{array}{c}\text { Sin Riesgo } \\
(\%)\end{array}$ \\
\hline Mares & 14 & 43 & 36 & 7 \\
Guanentina & 25 & 19 & 37 & 19 \\
García Rovira & 18 & 52 & 12 & 18 \\
Comunera & 63 & 32 & 5 & 0 \\
Soto & 32 & 50 & 9 & 9 \\
Vélez & 16 & 28 & 28 & 28 \\
Santander & 30 & 38 & 21 & 11 \\
\hline
\end{tabular}

Fuente: Tomado de "Situación Sanitaria de Santander", 2008

El uso de servicios mejorados de saneamiento es otro logro no alcanzado aún en Colombia, sólo el $74 \%$ de la población dispone de este servicio, lo que ubica al país en el puesto 23 a más de 10 puntos de la media de Latino América que es 87\%, y por debajo de un país con alto índice de pobreza como Guatemala (OMS, 2010).

En el departamento de Santander el porcentaje de la población que cuenta con este servicio es aún menor que el de todo el país, solo las provincias de Soto y Mares superan el $70 \%$.

Ante la carencia de alcantarillado, el pozo séptico es el segundo servicio alternativo más frecuente, seguido de la letrina sanitaria. La Tabla 6 da cuenta de los porcentajes de utilización de diferentes clases de servicios sanitarios.

Tabla 6. Clases de servicios sanitarios y su frecuencia en los hogares de Santander $\mathrm{y}$ en sus provincias

\begin{tabular}{lcccc}
\hline \multicolumn{5}{c}{ Clase de Servicio Sanitario } \\
\hline \multicolumn{1}{c}{ Provincias } & $\begin{array}{c}\text { Conectado al } \\
\text { alcantarillado }\end{array}$ & $\begin{array}{c}\text { Conectado } \\
\text { pozo séptico }\end{array}$ & $\begin{array}{c}\text { Sin } \\
\text { conexión }\end{array}$ & $\begin{array}{c}\text { Sin } \\
\text { sanitario }\end{array}$ \\
\hline Mares & 70,3 & 11,1 & 6,2 & 12,3 \\
Guanentina & 48,4 & 33,8 & 3,6 & 14,2 \\
García Rovira & 41,5 & 41,2 & 1,2 & 16,2 \\
Comunera & 43,4 & 27,2 & 10,6 & 18,7 \\
Soto & 85,3 & 8,9 & 2,4 & 3,4 \\
Vélez & 40,1 & 29,2 & 6,1 & 24,6 \\
Santander & 71,4 & 15,5 & 3,9 & 9,2 \\
\hline
\end{tabular}

Fuente: Cálculos realizados con base a datos del censo 2005 (DANE) 
La disposición de basuras es otro indicador que puede asociarse a la mayor frecuencia de enfermedades diarreicas debido principalmente a la contaminación de las aguas.

Sólo en Mares y Soto más del 70\% de las viviendas cuentan con sistema de aseo que recolecta las basuras. En el resto de las provincias, en más del 50\% de las viviendas la basura es enterrada, quemada y/o tirada al patio, ríos y caños (DANE, 2005).

Con respecto a la contaminación alimentaria, la Secretaría de Salud Departamental informó en el año 2008 que un 55\% de las muestras de alimentos analizadas contenían elementos que los clasificaban como de alto riesgo para su consumo.

De todos los alimentos la leche y sus derivados fue catalogada de alto riesgo para la salud pública $(43 \%)$ y no apta por sus características microbiológicas y fisicoquímicas.

Llama la atención que en el segundo lugar en la escala de riesgo se ubiquen los alimentos infantiles. El nivel de calidad de ciertos alimentos de la canasta básica familiar en Santander se muestra en la Tabla 7.

Tabla 7. Calidad de los alimentos según nivel de riesgo para su consumo.

\begin{tabular}{lccc}
\hline \multicolumn{4}{c}{ Clase de Servicio Sanitario } \\
\hline \multicolumn{1}{c}{ Alimentos } & $\begin{array}{c}\text { Alto Riesgo } \\
\text { Salud Pública }\end{array}$ & $\begin{array}{c}\text { No aptos Microbioló } \\
\text { gicamente }\end{array}$ & $\begin{array}{c}\text { No aptos Fisicoquí } \\
\text { micamente }\end{array}$ \\
\hline Leche y sus derivados & 43 & 48 & 86 \\
Alimentos infantiles & 22 & 21 & 7 \\
Agua envasada & 2 & 0 & 7 \\
Carne y productos cárnicos & 16 & 13 & 0 \\
Alimentos baja acidez & 15 & 14 & 0 \\
Alimentos a base de huevo & 2 & 3 & 0 \\
\hline
\end{tabular}

Fuente: datos tomados de "Situación Sanitaria de Santander", 2008

De todos los contaminantes, los coliformes totales y fecales son los más frecuentes, seguidos de esporas clostridium y mohos. 


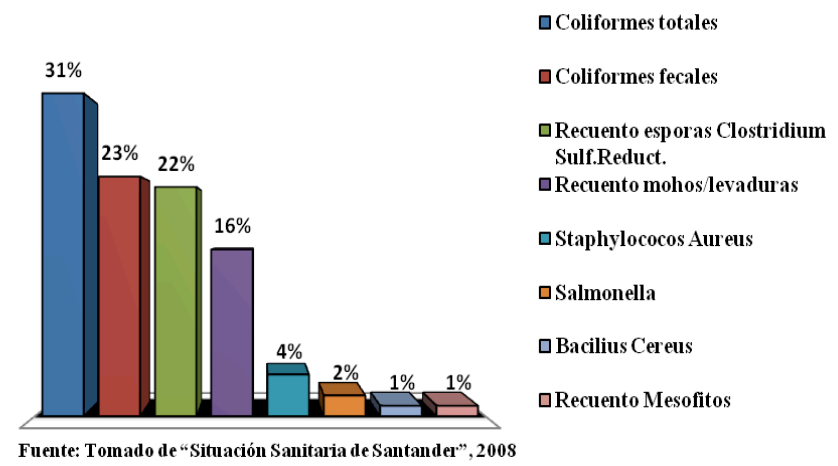

\section{Conclusiones}

En el departamento de Santander la población de niños menores de 12 años muestra un perfil de morbilidad característico de una región con altos niveles de pobreza, o en desarrollo, cuyas patologías son atribuidas a factores ambientales que pueden ser modificados principalmente de acuerdo a los problemas económicos de la población y para mejorar las condiciones sanitarias.

El importante desarrollo de la economía del departamento en los años recientes no ha beneficiado al total de la población: existe un elevado número de personas que viven en condiciones paupérrimas y un grupo de personas, aún mayor, en condiciones de pobreza, de lo que se deduce que la distribución de la riqueza en el departamento es altamente inequitativa.

Los beneficios económicos tampoco han contribuido al desarrollo de adecuados servicios sanitarios y a una producción de alimentos saludables, lo que podría explicar la alta prevalencia de enfermedades diarreicas y gastrointestinales por infección en los niños menores de 12 años.

La población rural y los residentes de las provincias de García Rovira y Vélez se constituyen en el grupo humano más propicio a sufrir enfermedades atribuibles a factores ambientales.

Mientras no se mejoren las condiciones sanitarias, la prevalencia de enfermedades diarreicas se mantendrá o se elevará con el consiguiente incremento de los gastos no sólo en salud si no también al interior de la familia, con el consiguiente deterioro de la calidad de vida.

Recomendación: Los antecedentes y los resultados de este estudio indican la necesidad de realizar investigaciones explicativas que den cuenta de la relación entre 
la exposición ambiental y la salud de la población, en especial la población infantil $\mathrm{y}$, con base a los resultados, diseñar e implementar intervenciones que den solución permanente a los problemas.

Entre tanto, como medida preventiva de las enfermedades diarreicas, las autoridades locales deben comprometerse a realizar las inversiones necesarias para permitir que toda la población tenga acceso a fuentes inocuas de agua de consumo, mejorar el saneamiento, realizar campañas educativas tendientes a fomentar ambientes saludables, mejorar los hábitos de higiene personal y manipulación de alimentos enfatizado los modos de transmisión de las infecciones.

\section{REFERENCIAS BIBLIOGRÁFICAS}

ACR. Alta Consejería para la Reintegración. (2008). Diagnóstico Socioeconómico Departamento de Santander. Bogotá D.C.

Cepeda, L. (2010). ¿Por qué le va bien a la economía de Santander? Documento de trabajo sobre Economía Regional. Número 135, Banco de la República. Centro de Estudios Económicos Regionales (CEER) - Cartagena. Recuperado el 6 de mayo 2012 del sitio Web: http://www.banrep.gov.co/documentos/ publicaciones/ regional/documentos/DTSER-135.pdf.

Chelala, C. Impacto del ambiente sobre la salud infantil. (1999). OPS, Washington, D.C. Recuperado el 8 de mayo 2012 del sitio Web: http://www.paho.org/ spanish/ hep/infancia.pdf.

DANE. Censo ampliado, 2005. Recuperado en febrero del 2011 del sitio Web: http:// www.dane.gov.co/daneweb.

DANE. Necesidades Básicas Insatisfechas. Recuperado el 1 de julio 2011 del sitio Web:http://www.dane.gov.co/daneweb_V09/index.php?option=com_content\&vie $\mathrm{w}=$ article\&id $=57 \&$ Itemid $=66$.

DANE. Perfil Departamental Santander. Boletín General. (2010). Recuperado en marzo 1 de julio 2010 del sitio Web:

http://www.dane.gov.co/daneweb_V09/index.php?option=com_content\&view=articl e\&id=57\&Itemid=66.

EHP-SPM. (2009). Noticias de salud ambiental. Environ Health Perspectiv, 117, 543 547.

Mardones - Restat, F. Díaz, M. Bonnefoy, JC. (1994). Una propuesta de clasificación de las comunas del país, según la situación de la infancia. Santiago, Chile: UNICEF. 
OMS Organización Mundial de la Salud. (2002). Informe sobre la salud en el mundo. Recuperado el febrero 2011 del sitio Web: http://www.who.int/whr/2002/en/ whr02_es.pdf.

OMS. Organización Mundial de la Salud. (2001). Protección y desarrollo ambiental. Recuperado el 8 de mayo 2012 del sitio Web: http://www.paho.org/ spanish/d/ DO302_03.pdf.

OMS Organización Mundial de la Salud. (2010). Estadísticas Sanitarias Mundiales. Recuperado el febrero 2011 del sitio Web: http:/www.who.int/whosis/whostat/ ES_WHS10_Full.pdf.

OMS Organización Mundial de la Salud. (1993). Nuestro planeta, nuestra salud: Informe de la Comisión de Salud y Medio Ambiente de la OMS. Washington DC: OPS; Publicación científica 544.

Ordóñez, G. (2000). Salud ambiental: conceptos y actividades. Rev. Panam Salud Pública, 7, $137-147$.

OSPS Observatorio de Salud Pública de Santander. (2006). Indicadores de Morbilidad basados en Registros Individuales de Prestación de Servicios (RIPS). Segunda Edición.

OSPS Observatorio de Salud Pública de Santander. (2009). Perfil de Morbilidad y Mortalidad en Santander de año 2007. Revista OSPS, Año 4, Número 2.

OSPS Observatorio de Salud Pública de Santander. (2010). Indicadores de Morbilidad basados en el Registro Individual de Prestación de Servicios de Salud (RIPS). Suplemento de Revista OSPS, Año 5, Número 2.

PDD. Plan de Desarrollo Departamental. 2008 - 2011. Gobernación de Santander. Ordenanza Nº 08 (Mayo 30 de 2008).

PDD. Plan de Desarrollo Departamental. 2012- 2015. Gobernación de Santander. Proyecto de Ordenanza.

PIB. Producto Interno Bruto por Departamento. Recuperado el 8 de mayo 2012 del sitio Web: http://www.sintramites.com/temas/indicadoresantander/indicadores/ pibxdptos.htm.

Pond, K. Kim, K. Carroquino, M. Pirard, P. Gore, F. Cucu, A. Nemer, L. MacKay, M. Smedje, G. Georgellis, A. Dalbokova, D. Krzyzanowski, M. (2007). Workgroup Report: Developing Environmental Health Indicators for European Children: World Health Organization Working Group. Environ Health Perspect, 115:13761382 . 
Prüss-Ustün, A. Corvalán, C. (2006). Preventing Disease Through Healthy Environments. Towards an Estimate of The Environmental Burden of Disease. Geneva: World Health Organization. Recuperado en febrero 2011 del sitio Web: http://www.who.int/quantifying_ehimpacts/publications/preventingdisease/en.

Ramírez, J. Osorio, H. Parra, R. (2007). Escalafón de la competitividad de los departamentos de Colombia. CEPAL, Serie Estudios Perspectiva, Volumen 16. Santiago, Chile: Naciones Unidas.

SIVILA. Sistema de Vigilancia Epidemiológica, Secretaría de Salud Departamental. Gobernación de Santander. (2010). Indicadores de Salud de Santander.

SSD Secretaria de Salud Departamental. (2008). Situación Sanitaria del Departamento de Santander.

SSD Secretaria de Salud Departamental. (2004). Diagnóstico de la Situación de Salud en Santander.

Valent, F. Little, D. Tamburlini, G. Barbone, F. (2004). Burden of disease attributable to selected environmental factors and injuries among Europe's children and adolescents. Geneva, World Health Organization, WHO Environmental Burden of Disease Series, No. 8. Recuperado el 8 de mayo 2012 del sitio Web: www.who. int/quantifying.../en/ebd8intro.pdf.

WHO. (2010). Fifth Ministerial Conference on Environment and Health Protecting children's health in a changing environment Parma, Italy, 10-12 March 2010. Recuperado el 6 de mayo 2012 del sitio Web:

http://www.euro.who.int/_data/assets/pdf_file/0004/104476/parma_leaflet.pdf

WHO. (2002). Report of the International Conference on Environmental Threats to the Health of Children: Hazards and Vulnerability. Bangkok, Thailand, 3-7 March, 2002. Geneva: World Health Organization. Recuperado el 8 de mayo 2012del sitio Web: http://www.who.int/docstore/peh/ceh/Bangkok/Bangkokconfreport. pdf.

WHO Regional Office for Europe. (2004). Fourth Ministerial Conference on Environment and Health, Budapest, Hungary, 23-25 June 2004. Recuperado el 8 de mayo 2012 del sitio Web: http://www.euro.who.int/document/e83335.pdf. 